\title{
Stress Relaxation in Shot-Peened Geometric Features Subjected to Fatigue : Experiments and Modelling
}

\author{
Mithila Achintha ${ }^{1, a^{*}}$, Chao You ${ }^{1, b}$, Binyan He ${ }^{1, c}$, Kath Soady ${ }^{1, d}$ \\ and Philippa Reed ${ }^{1, e}$
}

\author{
${ }^{1}$ Faculty of Engineering and the Environment, Univ. of Southampton, Southampton, SO17 1BJ, UK \\ aMithila.Achintha@soton.ac.uk, bcy4g13@soton.ac.uk, 'bh2g11@soton.ac.uk, \\ dK.A.Soady@soton.ac.uk and ${ }^{\mathrm{e} P . A . R e e d @ s o t o n . a c . u k ~}$
}

${ }^{*}$ Corresponding Author

Keywords: Eigenstrain, FE analysis, Residual Stress, Stress Relaxation

\begin{abstract}
This paper investigates experimentally and numerically the degree to which the stress relaxation in simple shot-peened geometries can be modelled by using a knowledge of simplified eigenstrain distributions. The residual stress generated due to shot peening is determined as the elastic response of the whole component when the plastic strains caused by the process are incorporated as an eigenstrain in an appropriate finite element (FE) model. The application of a subsequent live load is then modelled as an additional load step in the FE model which will superpose the effect of this loading on the residual stress field. The results show that the hybrid eigenstrain/FE approach is particularly useful in predicting residual stress relaxation in shot-peened components.
\end{abstract}

\section{Introduction}

Turbine blades of conventional power plants are mostly manufactured from ferritic heat resistant steels $[1,2,3]$, and are susceptible to damage and crack formation in regions of contact exposed to both centrifugal loading and oscillatory vibrations. One particular location where high stress concentrations are present and which is therefore susceptible to fatigue failure is the fir-tree blade root. In highly stressed areas, microcracks can initiate within the first few thousand cycles, significantly reducing the life time of the component. The propagation of microcracks under low cycle fatigue (LCF) may therefore eventually cause final fast failure. During a major outage, turbines may need to shut down for considerable periods causing significant financial loss for the energy supplier [1] and by extension to the wider economy.

Shot peening is usually applied to regions of stress concentration in steel turbine blades as a means of improving fatigue life. During the process of shot peening, a stream of small steel shots bombard the work surface by means of a compressed air jet. Each piece of shot that strikes the material acts as a tiny peening hammer. The kinematic energy of a shot impacting on the surface causes a small indentation or dimple which causes the material in the surface region to yield in tension. Once the shot rebounds from the surface, the mismatch between the plastically deformed surface zone and the elastically unloading bulk material underneath causes a compressive residual stress in the near surface region of the component. The counterbalancing tension is carried by the subsurface material underneath the peened area and/or outside the unpeened area. Typically, shot peening generates a surface compression layer of $\sim 0.2-0.4 \mathrm{~mm}$ thick with a peak surface compressive stress of about $\sim 60-70 \%$ of the monotonic yield stress of the material [4].

The surface compressive residual stress and associated strain hardening of the material are usually considered to increase the fatigue life since both retard the development and propagation of fatigue cracks. It should, however, be appreciated that the increased surface roughness caused by shot peening can actually reduce crack initiation time as a result of the presence of micro stress concentrators [1]. However, it has been determined that the beneficial effects of the surface compressive residual stress and strain hardening usually dominate any detrimental surface roughness 
effects and the overall effect of shot peening is to increase fatigue life $[1,3,4]$. Shot peening is particularly attractive for application at geometric stress concentrations. However, these are precisely the areas where the effect of shot peening is most difficult to predict: the interaction between the process and complex geometries can lead to unexpected results. The stresses generated during subsequent LCF can reach levels sufficient for further plastic deformation in the material, and subsequently, can diminish the beneficial effects of the original surface compression.

Several investigations into the relaxation of compressive residual stress after fatigue cycling are reported in the literature; albeit the majority of the works considered the relaxation of residual stresses in flat, axially loaded samples [1]. Although more geometrically complex samples representing stress concentration features such as notches are considered in some studies, they were mostly tested only under axial loads [1]. The literature provides some useful insights into how stress relaxation occurs in shot-peened metallic components, however, this is limited to experimental observations of the specific test parameters chosen in each case; more generalised prediction of stress relaxation for a different set of shot peening parameters or in different component geometries requires further validation.

Validated life assessment models are needed to allow understanding of the structural response of shot peened turbine materials under LCF, and thus to enable scheduling maintenance work, and/ or to prevent severe outages [1]. The difficulty that inhibits accurate prediction of the LCF response of shot peened components is the lack of a comprehensive analytical or numerical tool to predict residual stress distributions generated in different geometric features. It should be noted that residual stresses cannot be measured directly, rather they are deduced from measured residual elastic strains (usually by using X-ray or neutron diffraction techniques) using a suitable form of Hooke's law. These residual elastic strains are only measured at a finite number of measurement locations and determination of the full stress field from these measurements (i.e. six continuously varying components of stress that satisfy equilibrium, compatibility and the boundary conditions) is an extremely challenging problem, particularly in the presence of measurement error or uncertainty. There is a need for a more physically based model which represents the plastic strain introduced by the shot peening process, rather than seeking the residual stress field directly.

Once the plastic deformations caused by shot impacts are fully stabilised, the response of the workpiece is elastic. Therefore, the residual stress distribution can be determined as the elastic response of the workpiece if the stabilised plastic strains are incorporated as an initial misfit strain (i.e. an eigenstrain). The eigenstrain technique is an efficient tool to model the residual stress state present in a component and the technique has been successfully used for other applications: for instance, modelling residual stress generated due to laser shock peening [5-7]; stresses induced due to welding [8]; and fibre scales residual stress variation in metal-matrix composites [9]; and etc. The current paper exploits a hybrid eigenstrain/FE approach to model residual stress relaxation in shot-peened components. This is achieved by first incorporating plastic strain generated as an initial misfit strain in an appropriate elastic FE model. The implicit FE model includes the effect of shot peening as a misfit strain, and the residual stress arises as a response to this. Hence, application of a subsequent live load as an additional load step in the FE model will superpose the effect of this loading on the residual stress field [6]. This paper investigates experimentally and numerically the degree to which the LCF of shot-peened simple plain bending geometries can be modelled by using an understanding of simple eigenstrain distributions. The results suggest that the eigenstrain approach is particularly useful in predicting residual stress relaxation in shot-peened components.

\section{Eigenstrain Modelling of Residual Stress}

Modelling the residual stress field as an eigenstrain distribution is an essential prerequisite to analyse the stress relaxation during LCF. The approach has the benefit that the eigenstrain appears to be more characteristic of the shot peen process and it is largely independent of the component geometry whereas the residual stress field is influenced by the exact geometry of the work piece. Hence, the method may provide an attractive approach for investigating residual stress relaxation in different geometries and under different applied loading schemes. All that is required is a 'library' of 
different eigenstrain depth profiles caused by the application of a range of shot peen parameters to a single patch on a flat plate.

To illustrate the application of the model, the analysis of a simple four-point bend specimen of FV448 with dimensions $40 \mathrm{~mm} \times 8 \mathrm{~mm} \times 7.75 \mathrm{~mm}$ (Fig. 1), shot peened by a commercial service provider is discussed below. The details of the shot peening process parameters are: process M1230R 13A 200\% (T0); intensity - 13A; coverage - 200\%; shot diameter - $0.58 \mathrm{~mm}$; shot hardness $-45-52 \mathrm{HRC}$; and shot velocity $-57 \mathrm{~ms}^{-1}$. In order to remove any artefacts from the prior machining of samples by electrical discharge machining (EDM), $0.25 \mathrm{~mm}$ was removed from the surface by a grinding process, so that the surface roughness met the industrial machined component (and pre-peen) specification of $R_{a}<0.8 \mu \mathrm{m}$.

FV448 was chosen because of its widespread use in low pressure turbine blades [1]. The residual stresses were measured using a Proto iXRD device and instrumental layer removal was achieved by an electropolishing method as detailed in [1]. Residual stress data was collected in the two orthogonal directions $\left(\sigma_{x x}\right.$ and $\left.\sigma_{y y}\right)$ in the shot peened sample in the uncycled condition and after one loading cycle. Table 1 presents measured $\sigma_{x x}$ and $\sigma_{y y}$ stress values with depth $(z)$ from the peened surface. It can be seen from Table 1 that a compressive zone of $\sim 0.3-0.35 \mathrm{~mm}$ deep is developed underneath the peened surface with counter-balancing tensile stresses beneath. A compressive stress of $\sim 600 \mathrm{MPa}$ is developed at the surface which diminishes gradually and becomes tensile after $\sim 0.3-0.35 \mathrm{~mm}$ depth.

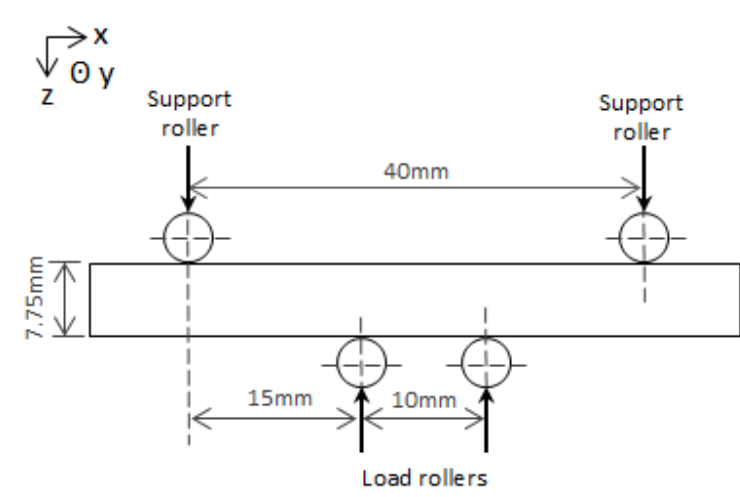

Fig. 1 - Specimen geometry
Table 1: Measured $\sigma_{x x}$ and $\sigma_{y y}$ with $z$

\begin{tabular}{c|c|c}
\hline Depth, $z(\mathrm{~mm})$ & $\sigma_{x x}(\mathrm{MPa})$ & $\sigma_{y y}(\mathrm{MPa})$ \\
\hline 0 & -594.4 & -546.4 \\
0.022 & -615.9 & -581.4 \\
0.047 & -618.6 & -549.5 \\
0.070 & -604.7 & -548.7 \\
0.096 & -598.6 & -579.7 \\
0.142 & -537.2 & -523.1 \\
0.170 & -510.0 & -508.3 \\
0.210 & -473.8 & -479.3 \\
0.244 & -368.4 & -375.8 \\
0.294 & -127.1 & -164.2 \\
0.364 & 52.8 & 50.3 \\
0.420 & 79.6 & 80.9 \\
\hline
\end{tabular}

The objective of the current analysis is to determine an accurate estimate for the experimental eigenstrain distribution by using the knowledge of known residual stresses at different depths - i.e. an inverse eigenstrain analysis. This is conveniently achieved by first assuming a sensible choice of a parametric form for the experimental eigenstrain distribution (e.g. a polynomial distribution over a given depth), and then by matching the residual stresses predicted when this assumed eigenstrain distribution is incorporated in a FE model with the corresponding measured stresses in a least squares sense. Once an accurate estimate for the experimental eigenstrain distribution has been established, the complete residual stress field in the whole component can be determined in the usual way by incorporating this correct eigenstrain distribution in an FE model [6]. The advantage of this approach is that the elastic response of the workpiece to the eigenstrain will satisfy equilibrium, compatibility and boundary conditions, and the residual stress field produced will be entirely self-consistent.

It is anticipated that the material will experience high strain rates during shot peening. In such circumstances, relatively sophisticated material models, which incorporate strain and strain rate hardening, may appear to give a more appropriate representation of material behaviour. However, in practice the material parameters required are very difficult to determine and they can simply obscure the underlying physical behaviour without giving a significant increase in fidelity. The focus of current analysis is to establish an accurate estimate for the eigenstrain distribution which can 
represent the measured residual stress distribution. In such circumstances, it is reasonable to employ a linear elastic model with properties broadly representative of FV448 (Young's modulus $(E) 201.3$ $\mathrm{GPa}$; Poisson's ratio (v) 0.3) in this inverse eigenstrain analysis. However, it should be appreciated that a material model that includes strain hardening effects will be used in the subsequent stress relaxation analysis.

The analysis requires the choice of a suitable parametric form for the eigenstrain distribution. A method based on Chebyshev polynomials will be adopted here [6], although alternative choices are possible. The inverse eigenstrain analysis is started by first assuming that the eigenstrain distribution $\left(\varepsilon_{x x}^{p}\right)$ may be represented as a Chebyshev series of $N$ polynomials [6]. The choice of $N$ is to some extent arbitrary although it should be less than the total number of stress measurements. Similarly, it should be large enough to capture the expected form of the eigenstrain distribution with acceptable accuracy. In practice, the analysis can be carried out for a number of different $N$ values and a check will be carried out to ensure that the results are largely independent of the value chosen. In the current example, it was determined that any $N \geq 6$ gives accurate results. It is also necessary to choose the depth of the eigenstrain distribution at the beginning of the inverse eigenstrain analysis; it has been shown that [6] as long as this choice is sensible, the answer is largely independent of the choice. The measured residual stresses (Table 1) indicate that the effects of shot peening diminishes after 0.3-0.35 $\mathrm{mm}$; thus the inverse eigenstrain analysis was carried out by assuming the depth of eigenstrain distribution as $0.4 \mathrm{~mm}$. Details of a comprehensive least squares analysis of an inverse eigenstrain analysis can be found in elsewhere [6] and is not repeated here.

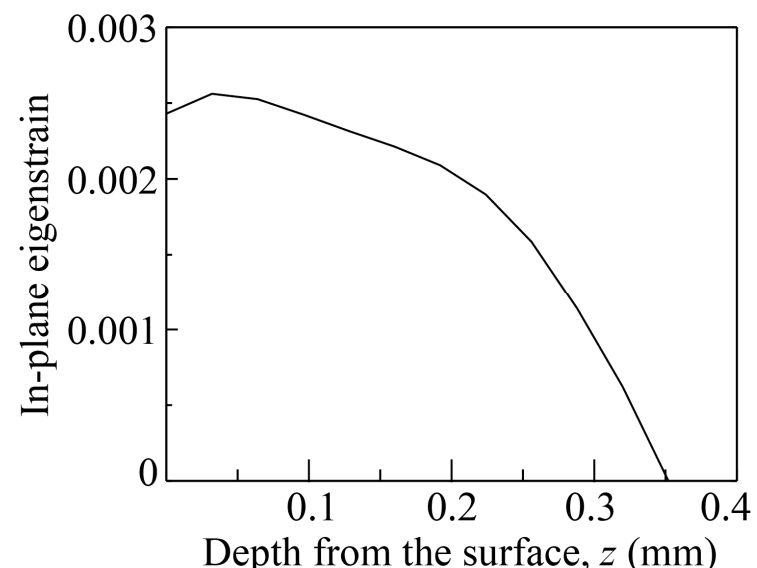

Fig. 2a - Variation of in-plane eigenstrain component $\varepsilon_{x x}^{p}$ with depth from the surface

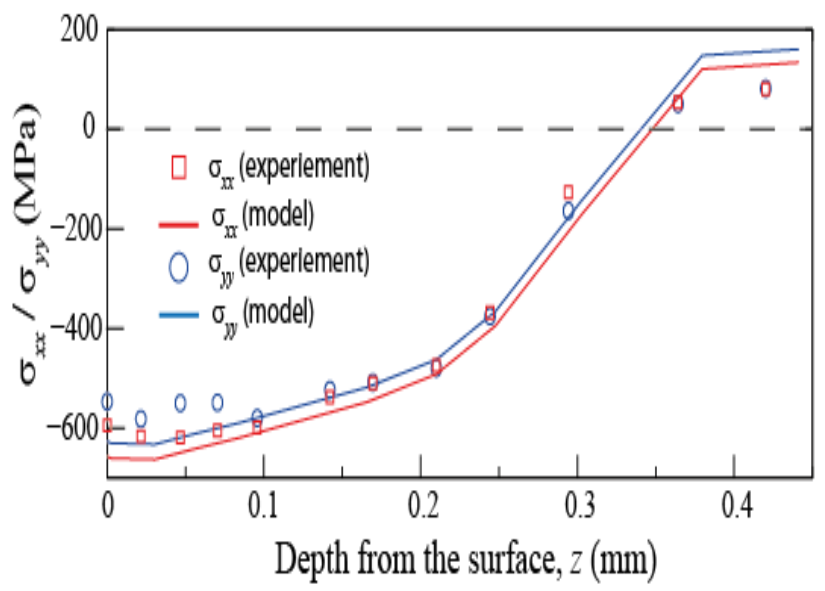

Fig. $2 \mathrm{~b}$ - Comparison between the predicted and the measured residual stress data

Fig. 2a shows the estimated depth profile of one of the in-plane components of the eigenstrain $\varepsilon_{x x}^{p}$. The other two principal components of the eigenstrain may be determined by applying considerations of symmetry and of volume conservation in plastic deformation $\left(\varepsilon_{y y}^{p}=\varepsilon_{x x}^{p}\right.$ and $\left.\varepsilon_{z z}^{p}=-2 \varepsilon_{x x}^{p}\right)$. The results show that $\varepsilon_{x x}^{p}$ at the surface is $\sim 0.0025$ and it decreases to zero at $\mathrm{z} \approx 0.35 \mathrm{~mm}$. It is worth noting that the analysis was carried out with an assumed eigenstrain depth of $0.4 \mathrm{~mm}$, however, the results show that it is actually $\sim 0.35 \mathrm{~mm}$. From this result it is evident that the actual eigenstrain distribution is largely independent of the initially assumed depth. In order to conveniently incorporate the eigenstrain as a misfit strain in an appropriate FE model, it is useful to represent the variation of $\varepsilon_{x x}^{p}$ with $z$ approximately as a polynomial function of $z$. It was determined that this can be conveniently approximated by a $7^{\text {th }}$ order Chebyshev series within the depth range $0 \leq z \leq 3.5 \mathrm{~mm}$ (coefficients of the polynomial are $0.00240001,-0.0039856,0.00638822,-0.0093786,0.0079579,-0.0041980$, $0.0015399,-0.0000068$ ).

The distribution of all three principal components of the eigenstrain with depth was applied over the required volume in an elastic FE model (ABAQUS/Standard is used in the present study). The solution provides all six components of stress within the whole component that satisfy equilibrium, 
compatibility and boundary conditions. Fig. $2 \mathrm{~b}$ shows the variation of $\sigma_{x x}$ and $\sigma_{y y}$ with $z$ at the centre region of the peened area. The respective measured residual stress data are also presented in the figure. From the results shown in Fig. $2 \mathrm{~b}$ it is evident that the eigenstrain method accurately predicts both the $\sigma_{x x}$ and $\sigma_{y y}$ stress distributions. Similarly, a good agreement with experimental results was shown for another specimen treated with a shot peening process of lower intensity; because of space limitations the results of that analysis are not presented here.

\section{Modelling of Stress Relaxation}

The implicit FE model described in the previous section includes the effect of shot peening as a misfit strain, and the residual stress arises as response to this. The application of a subsequent live load due to LCF can then be modelled as an additional load step in the FE model which will superpose the effect of this loading on the residual stress. To illustrate the application of the method, the analysis of the same four-point bend specimen (Fig. 1) under one load cycle is discussed below.

In previous experiments [3], four-point bend fatigue loading was carried out with load ratio $R_{p}=$ 0.1 and peak load $P_{\max }=16794 \mathrm{~N}$, using a sinusoidal waveform of frequency of $20 \mathrm{~Hz}$. In the FE simulation, the same load values were applied, but a triangular waveform was chosen. In this experiment, a nominal stress $\left(\sigma_{\text {nom }}\right)$ is used to represent the stress level as defined by Eq. 1 (in Eq. $1, B$ $($ width $)=8 \mathrm{~mm} ; W($ depth $)=7.75 \mathrm{~mm}$; and $s$ (shear span) $=15 \mathrm{~mm}$ are beam dimensions (Fig. 1)). Under the loading arrangement considered in the study, $\sigma_{\text {nom }}=1523.3 \mathrm{MPa}$.

$$
\sigma_{\text {nom }}=\frac{3 s P_{\max }}{B W^{2}}
$$

The objective of the current analysis is to model the response after only one load cycle, based on previous experimental work for this material [10] we have therefore assumed an isotropic hardening material model in the analysis since the material will not undergo significant repeated cyclic behaviour. It has been shown [10] that the Ramberg-Osgood model (Eq. 2) satisfactorily represents the material behaviour of FV448 under monotonic loading, and this model is used in the present analysis. The associated calibration parameters determined from the tensile experimental data are: $E$ (Young's modulus) $=201.3 \mathrm{GPa}$, monotonic strain hardening exponent $n_{m}=0.05865$ and strength coefficient $A=1152 \mathrm{MPa}$ [10]; and is considered satisfactory for the purpose of validating the approach.

$$
\varepsilon=\sigma / E+(\sigma / A)^{\frac{1}{n_{m}}}
$$

Fig. 3 shows the evaluation of the strain hardening effect caused by shot peening. Previously EBSD based approaches [2] have been carried out to measure the distribution of plastic strain which was then used to determine the local strain hardening via implementation of a local modified $0.2 \%$ proof stress based on the Ramberg-Osgood relationship in Eq. 2. To incorporate the experimentally determined strain hardening effect, varying local material models were assigned to different depths in the FE model. The process of determining each material model is illustrated in Fig. 4, firstly the elastic regime is connected to the local yield point (zero plastic strain point), which is then connected to the $0.2 \%$ proof stress point using an approximated curve. Finally, the obtained stress-plastic strain curve is appropriately shifted to the origin of the coordinate system. This yield point is estimated as $82 \%$ of the corresponding $0.2 \%$ proof stress based on the $\sigma-\mathcal{E}$ response observed experimentally in the tested material. During calculation, ABAQUS automatically interpolates between the material models assigned at each depth, thus the whole hardened region can be achieved in the FE model. The material models at different depths are shown in Fig. 5. 


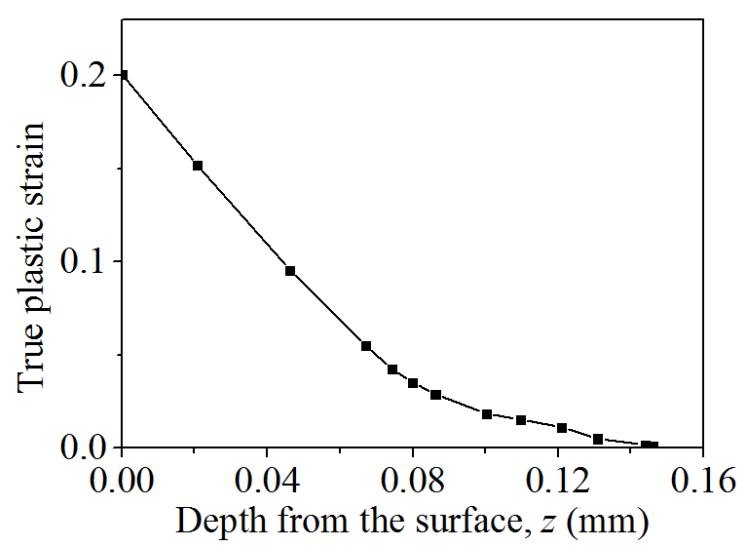

Fig. 3a - Plastic strain distribution in the shot peened sample measured by EBSD method [2]

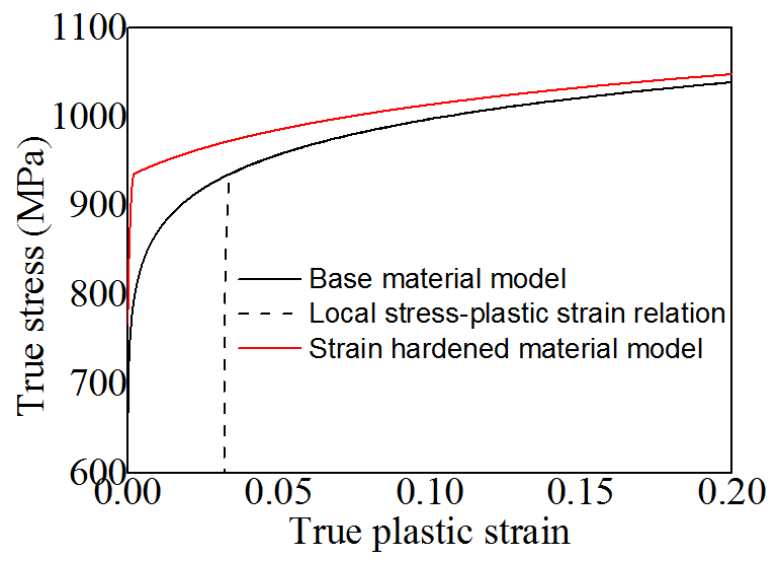

Fig. 4 - Determination of the strain hardened model

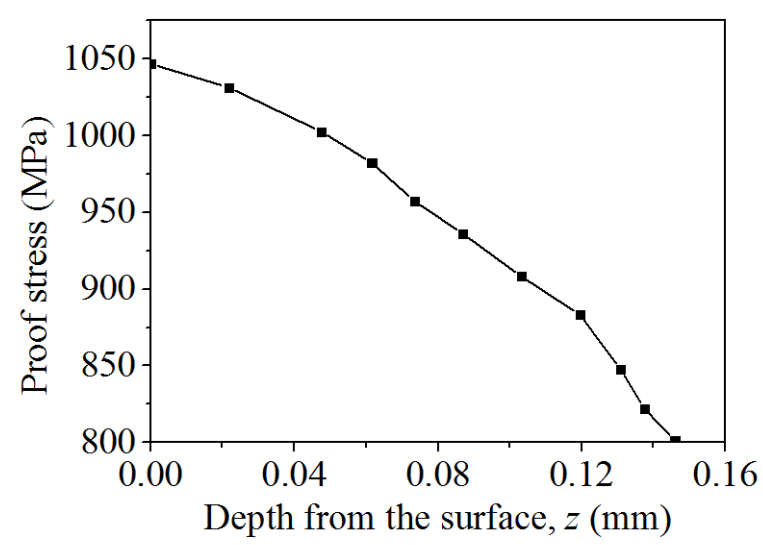

Fig. $3 b-$ Corresponding $0.2 \%$ proof stress distribution calculated using the Ramberg-Osgood relationship [2]

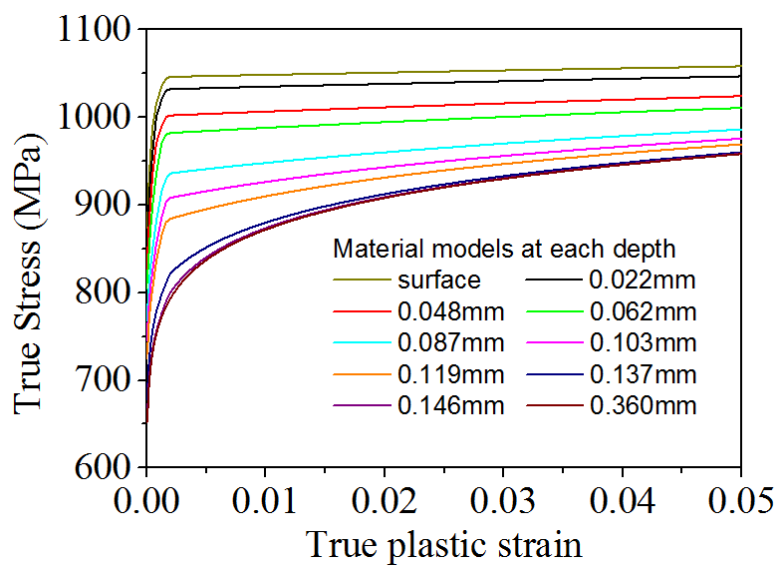

Fig. 5 - Material models at different depths

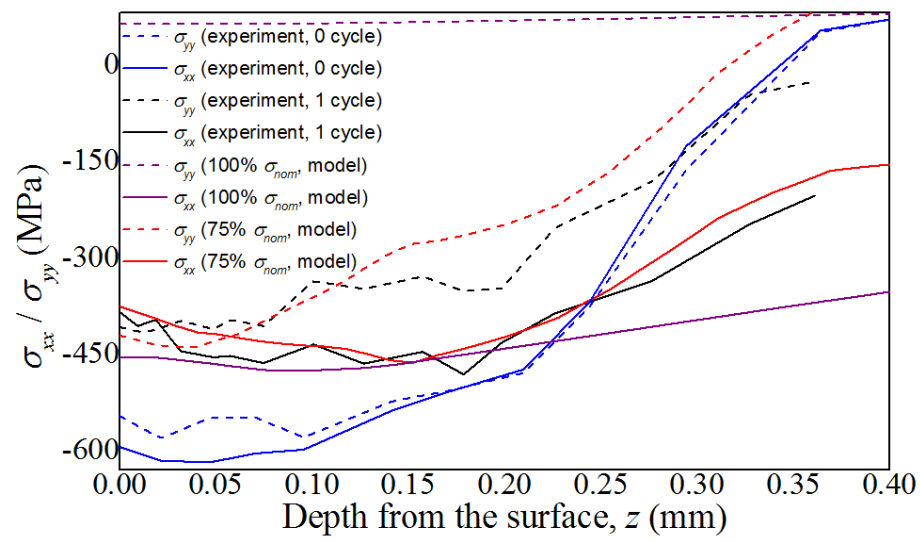

Fig. 6 - Comparison between experimental and simulated results of residual stress relaxation

Fig. 6 shows a comparison between experimental and simulated results of residual stress relaxation after one load cycle. It is found that when simulating the same nominal stress level in the $\mathrm{FE}$ as expected in the experiment $\left(\sigma_{\text {nom }}=1523.3 \mathrm{MPa}\right)$, the subsequent observed $\sigma_{y y}$ deviates significantly from the experimental data (with very much more stress relaxation predicted than is observed). However, applying a reduced stress level in the FE simulation, e.g. $0.75 \sigma_{\text {nom }}$, the simulated distribution of residual stress in both directions matches well with experimental results. This discrepancy may be partially explained by the choice of boundary conditions in the FE simulation. Experimental measurements of the strain achieved in a repeated single loading 
experiment of an un-shot-peened component indicated that a lower total strain was achieved experimentally than predicted by the current FE simulation. Further work is required to validate both the boundary conditions of the loading in the FE simulation, the actual loading achieved in the single fatigue cycle applied experimentally and the appropriate choice of an elasto-plastic material model to satisfactorily assess the level of stress relaxation occurring.

\section{Conclusions}

The study has shown that the eigenstrain finite element modelling approach works well to model residual stresses generated due to shot peening. The technique may be used to construct the full residual stress field in a component that satisfies equilibrium and compatibility conditions, based on measured residual elastic stresses at a finite number of measurement locations. Once the eigenstrain corresponding to a particular shot peening treatment is known, relaxation of the residual stress during subsequent loading can be calculated in a computationally efficient manner. Although further work is required to validate both the loading condition assumptions and elasto-plastic material model used, qualitatively we are able to predict stress relaxation after shot peening using this approach. The stress relaxation analysis in complex geometries such as that in notched bend specimens using the eigenstrain analysis are currently being undertaken and will be published in later work.

\section{Acknowledgements}

Financial support from China Scholarship Council (CSC) is gratefully acknowledged. M. Achintha wishes to thank Prof. David Nowell (University of Oxford) for his guidance when the first author started his work on eigenstrain methods. Thanks must also be extended to Gary Harrison at the University of Manchester, for his assistance in collecting residual stress data, and to E.ON New Build and Test for materials supply and support for the experimental programme.

\section{References}

[1] K.A. Soady, B.G. Mellor, J. Shackleton, A. Morris and P.A.S. Reed, The effect of shot peening on notched low cycle fatigue, Materials Sciences and Engineering A. 528(2011) 8579-8558.

[2] K.A. Soady, B.G. Mellor, G.D. West, G. Harrison, A. Morris and P.A.S. Reed, Evaluating surface deformation and near surface strain hardening resulting from shot peening a tempered martensitic steel and application to low cycle fatigue, Int. J. Fatigue 54 (2013) 106-117.

[3] B. Y. He, K. A. Soady, B. G. Mellor, A. Morris and P. A. S. Reed, Effects of shot peening on short crack growth rate and resulting low cycle fatigue behaviour in low pressure turbine blade material, Materials Science and Technology, 29(7) 788-796.

[4] M.A.S. Torres and H.J.C. Voorwald, An evaluation of shot peening, residual stress and stress relaxation on the fatigue life of AISI 4340 steel, Int. J. Fatigue. 24(2002) 877-886.

[5] M. Achintha and D. Nowell, Eigenstrain modelling of residual stresses generated by laser shock peening. J. Mater. Proces. Techno., 211(2011) 1091-1101.

[6] M. Achintha, D. Nowell, K. Shapiro and P.J. Withers, Eigenstrain modelling of residual stress generated by arrays of Laser Shock Peeing shots and determination of the complete stress field using limited strain measurements. Surf. Coat. Technology 216 (2013) 68-77.

[7] M. Achintha, D. Nowell, D. Fufari , L. Sackett and M. Bache, Fatigue behaviour of geometric features subjected to laser shock peening: experiments and modelling. Int. J. Fatigue (2013) doi:10.1016/j.ijfatigue.2013.04.016.

[8] A.M. Korsunsky, G.M. Regino and D. Nowell, Variational eigenstrain analysis of residual stresses in a welded plate, Int. J. Solids Struct. 44 (2006) 4574-4591.

[9] M.B. Prime and M.R. Hill, J. Measurement of fiber-scale residual stress variation in a metalmatrix composite, Compos, Mater. 38 (2004) 2079-2095.

[10] K.A. Soady, Reducing conservatism in life assessment approaches: Industrial stream turbine blade to disc interfaces and the shot peening process. EngD Thesis, Univ. of Southampton (2013). 